\title{
APPLICATION OF RF (40.68 MHZ) ARC DISCHARGE FOR PLASMA CHEMICAL CONVERSION OF VOLATILE CHLORIDES OF SILICON AND GERMANIUM
}

\author{
R. Kornev ${ }^{a, *}$, P. Sennikov ${ }^{a}$, V. Nazarov ${ }^{a, b}$, A. Sukhanov ${ }^{a}$, \\ L. ShaBAROVA ${ }^{a}$ \\ ${ }^{a}$ G.G. Devyatykh Institute of Chemistry of High-Purity Substances of RAS, 49 Tropinin Str., Nizhny Novgorod, \\ 603951, Russia \\ ${ }^{b}$ Institute of Applied Physics of RAS, 46 Ulyanova Str., Nizhny Novgorod, 603950, Russia \\ * kornev@ihps.nnov.ru
}

\begin{abstract}
The processes of hydrogen reduction of silicon and germanium chlorides are studied under $\mathrm{RF}$ conditions $(40.68 \mathrm{MHz})$ of contracted atmospheric pressure arc discharge stabilized between two rod electrodes. The main gas-phase and solid products of plasma-chemical transformations are determined. Using the hydrogen reduction of $\mathrm{SiCl}_{4}$ as an example, the numerical simulation of gas-dynamic and heat exchange processes for this type of discharge was performed. The studies were carried out for the optimal conditions for obtaining trichlorosilane using modern computational technologies of fluid dynamics which made it possible to detail the velocity and temperature fields, the gas flow rates in the plasma region, and also determine the main temperature zones affecting the hydrogen reduction process.
\end{abstract}

Keywords: RF arc discharge, reduction of volatile chlorides, simulation of gas-dynamic.

\section{Introduction}

Silicon and germanium remain the most popular material for microelectronics, photovoltaics and optics. The technologies of their industrial production, based on the synthesis and purification of their chlorides, require constant improvement in connection with the increased requirements for the environmental safety of production. Thus, for silicon tetrachloride, there is a need to process it into chlorosilanes - the initial products of the synthesis of silicon. The production of germanium from its tetrachloride is characterized by multistage which leads to an additional flow of impurities into the target product. In this regard, the study of the applicability of plasma-chemical technologies for the direct synthesis of silicon and germanium from $\mathrm{SiCl}_{4}$ and $\mathrm{GeCl}_{4}$ is an actual task. In the development of plasma methods, much attention is paid to the diagnosis of reactive plasma, which allows determining the gas temperature, classifying intermediate particles and establishing the mechanism of chemical reactions [1]. In some cases, when the plasma zone is small or unavailable for physical diagnostic methods, information about the temperature and the basic mechanisms of chemical reactions can be obtained by examining the gas-dynamic and heat exchange features of the process $[2,3]$. One of the ways to study gas-dynamic and heat exchange processes is their numerical simulation [4-6]. The purpose of this work was to study the influence of the main technological parameters of the process of hydrogen reduction of $\mathrm{SiCl}_{4}$ and $\mathrm{GeCl}_{4}$, such as pressure and the ratio of reagents on the degree of conversion, as well as an estimate of the gas temperature in the gas discharge zone using computational fluid dynamics.

\section{Experimental}

Experiments on the investigation of the reduction processes of silicon and germanium tetrachlorides in hydrogen plasma were carried out on installation which schematic diagram was described in detail in $[7,8]$. The power of high-frequency oscillator was $340 \mathrm{~W}$, the frequency was $40.68 \mathrm{MHz}$. The flow of plasmaforming gas $\mathrm{H}_{2}+\mathrm{SiCl}_{4}\left(\mathrm{GeCl}_{4}\right)$ was varied in the range of $100-700 \mathrm{~cm}^{3} / \mathrm{min}$. The pressure during the experiment was kept constant at 760 Torr. The molar ratio $\mathrm{H}_{2} / \mathrm{SiCl}_{4}\left(\mathrm{GeCl}_{4}\right)$ was changed in the range of $2-15$. The plasma-chemical reactor was a tube of silica glass, along the axis of which the electrodes were placed. A high-frequency voltage was applied to the electrodes from the generator through a matching device and the discharge was ignited between the electrodes. A vapor-gas mixture of silicon tetrachloride and hydrogen was supplied to the discharge volume. The power supplied to the plasma discharge zone was determined by the calorimetric method according to the method [9]. The dependence of the yield of chlorosilanes and chlorogermans as well as silicon and germanium on the conditions of plasma-chemical reduction was experimentally investigated. The yield of silicon and germanium was determined by the gravimetric method with an accuracy of $1 \cdot 10^{-4} \mathrm{~g}$. The content of chlorosilanes and chlorogermanes was determined by gas chromatography with the detection limit of $0.1 \%$ and IR spectroscopy with a resolution of $0.1 \mathrm{~cm}^{-1}$. 


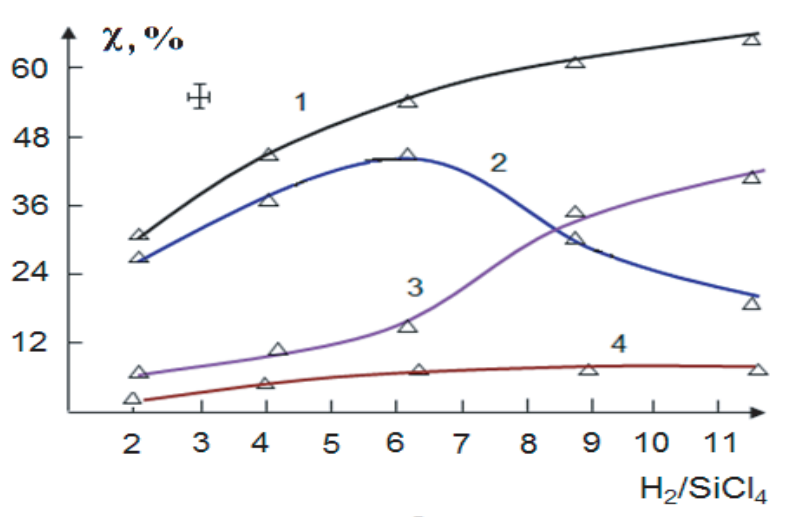

a

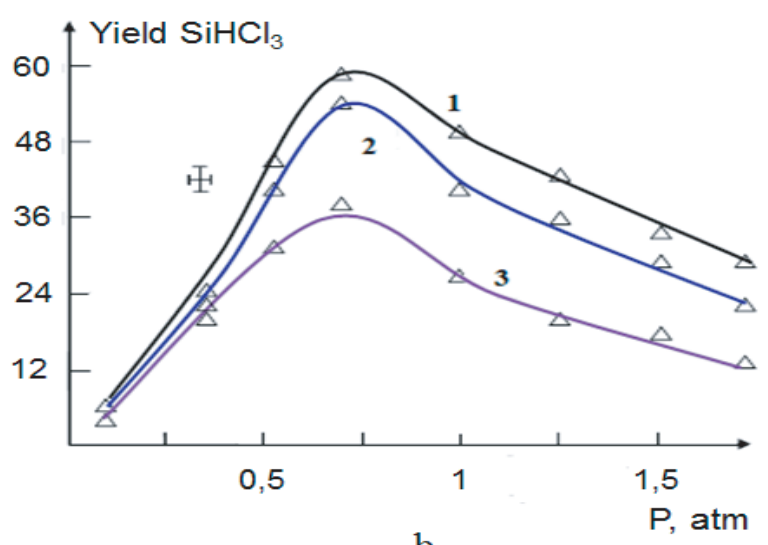

$\mathrm{b}$

Figure 1. a is the dependence of the total degree of conversion of $\mathrm{SiCl}_{4}(1)$, the percentage of yield of $\mathrm{SiHCl}_{3}$ (2), $\mathrm{Si}$ (3) and $\mathrm{SiH}_{2} \mathrm{Cl}_{2}$ (4) on $\mathrm{H}_{2} / \mathrm{SiCl}_{4}$ ratio; $b$ is the dependence of the yield of $\mathrm{SiHCl}_{3}$ on the pressure in reactor, $\mathrm{H}_{2} / \mathrm{SiCl}_{4}=6-(1), 4-(2), 9-$ (3).

\section{Mathematical Model}

The mathematical model was built in homogeneous formulation of the problem and included five different media: $\mathrm{H}_{2}, \mathrm{SiCl}_{4}$, air - liquids and solids - silicon and silica. The thermophysical and transport characteristics of these media are taken from $[10,11]$. The reactor was a silica tube with a diameter $60 \mathrm{~mm}$ where nozzleselectrodes were coaxially arranged to meet each other. The diameter and length of the nozzle $10 \mathrm{~mm}$ and $50 \mathrm{~mm}$, the length of the end portion of the nozzle is $15 \mathrm{~mm}$. The diameter and length of the electrode $6 \mathrm{~mm}$ and $70 \mathrm{~mm}$, the distance to which the electrode leaves the nozzle is $10 \mathrm{~mm}$. The distance between the electrodes is $7 \mathrm{~mm}$, the diameter of the outlet of the reactor is $10 \mathrm{~mm}$. The problem of heat transfer in the hydrodynamic flow of a mixture of ideal gases is considered in the mathematical formulation. The mode of flow of gases is turbulent. The mixture interacting in the flow is considered taking into account heat transfer by means of convection and heat conduction and is described by the following system of partial differential equations:

$$
\frac{\partial \rho_{i}}{\partial t}+\operatorname{div}\left(\rho_{i} \vec{V}_{i}\right)=0
$$

$$
\begin{array}{r}
\frac{\partial\left(\rho_{i} \vec{V}_{i}\right)}{\partial t}+\nabla\left(\rho_{i} \vec{V}_{i} \otimes \vec{V}_{i}\right)=-\nabla p+\nabla \tau_{i}, \\
\frac{\partial\left(\rho_{i} c_{p i} T\right)}{\partial t}+\operatorname{div}\left(\rho_{i} c_{p i} T \vec{V}_{i}\right)= \\
=\operatorname{div}\left(\lambda_{i e} \nabla T\right)+\operatorname{div}\left(\vec{V}_{i} \tau_{i}\right)+\frac{\partial p}{\partial t}+S_{E},
\end{array}
$$

where index $i=1$ refers to $\mathrm{H}_{2}, i=2$ - to $\mathrm{SiCl}_{4}$, $i=3$ - to air, $i=4$ - to silica, $p$ is the absolute pressure, $T$ is the absolute temperature, $\rho_{i}$ is the density of corresponding environment, $\rho_{1}, \rho_{2}$ are calculated from the equation of ideal gas, $\rho_{3}, \rho_{4}$ are constants, $\vec{V}_{i}$ is the velocity vector of corresponding environment, $S_{E}$ is the power induced by discharge, $\tau_{i}$ is the stress tensor connected with the rates of deformation generalized by Newton/s hypothesis $\left.\tau_{i}=\mu_{i e}\left(\operatorname{div}\left(\vec{V}_{i}\right)+\operatorname{div}\left(\vec{V}_{i}\right)\right)^{\mathrm{T}}-\frac{2}{3} \delta \operatorname{div}\left(\vec{V}_{i}\right)\right), \mu_{i e}, \lambda_{i e}$ are effective viscosity and thermal conductivity of environment, correspondingly, $\mu_{i e}=\mu_{i}+\mu_{i t}, \lambda_{i e}=\lambda_{i}+\lambda_{i t}$, $\mu_{i}, \lambda_{i}$ are the laminar viscosity and thermal conductivity, $\mu_{i t}, \lambda_{i t}$ are turbulent (vortex) viscosity and thermal conductivity which are interconnected through the turbulent Prandtl number and determined by the chosen model of turbulence [12]. The plasma region was a ball with a radius of $R / 2 \approx 7 \mathrm{~mm}$ in contact with the electrodes. The temperature of the electrode in the places of their contact with the plasma is set at $\approx 1760 \mathrm{~K}$. To simulate the plasma, an energy source is created in the reactor. The region of energy release is a cylinder at the base of which a circle with a radius of $P=1.375 \mathrm{~mm}$ and a height $H=2.5 \mathrm{~mm}$. The density of energy release is given by the formula

$$
\left.q=10^{9}\left(1-8 \cdot 10^{5}(h+H)^{2}\right) \pi P^{2}\right),
$$

where $h$ is the cylinder height.

The density of energy release is set thus so that the total value in the field of energy release corresponds to $15 \mathrm{~W}$. For silica walls of the reactor, only the energy equation is solved which reduces to the heat conduction equation for solid walls. Note that the gases in the problem are considered to be the compressible liquids. The system of equations (1)-(3) is closed by the transport SST model of Menter turbulence [13]. This model is a combination of the $k-\omega$ model (a more accurate description of flows near the walls) and the $k-\varepsilon$ model (simulation of currents far from solid boundaries).

\section{Discussion of the results}

The dependence of the degree of conversion of $\mathrm{SiCl}_{4}$ to chlorosilanes and silicon on the ratio of reagents in the initial mixture is shown in Figure 1a. The maximum yield of $\mathrm{SiHCl}_{3}(44 \%)$ is observed when the ratio $\left[\mathrm{H}_{2}\right]:\left[\mathrm{SiCl}_{4}\right]=6.9$. With further increase in the concentration of hydrogen, the yield of trichlorosilane decreases. The yield of Si with increasing hydrogen concentration monotonously increases from 8 to $47 \%$, and $\mathrm{SiH}_{2} \mathrm{Cl}_{2}$ from 3 to $8 \%$. It should be noted that the results on the yield of trichlorosilane (Fig. 1a) 


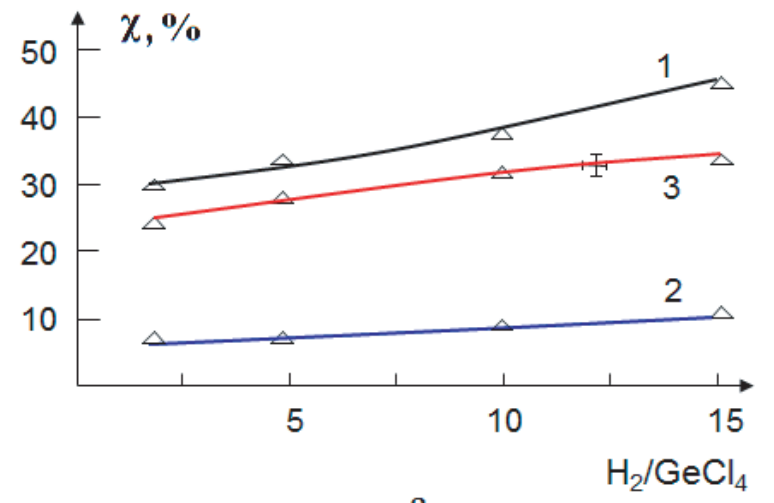

a

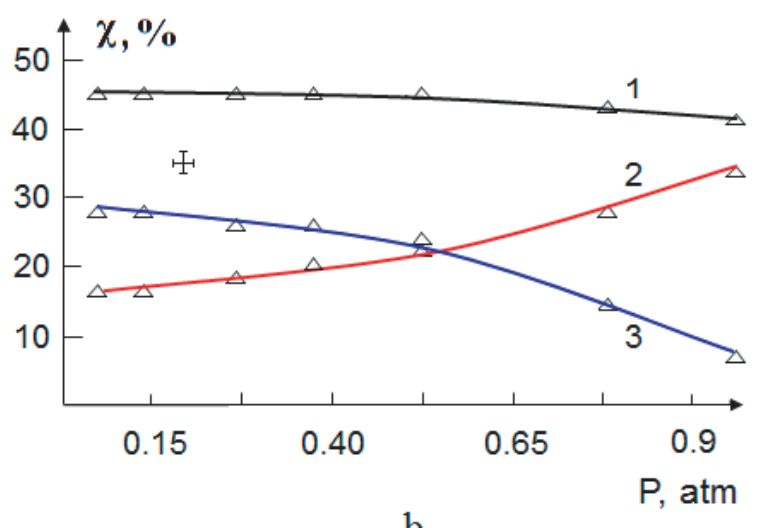

b

Figure 2. a is the dependence of the total degree of conversion of $\mathrm{GeCl}_{4}$ (1) and percentage yield of $\mathrm{Ge}$ (2) and $\mathrm{GeHCl}_{3}$ (3) on $\mathrm{H}_{2} / \mathrm{GeCl}_{4}$ ratio; $b$ is the dependence of the total degree of conversion of $\mathrm{GeCl}_{4}$ (1) and the percentage yield of $\mathrm{GeHCl}_{3}$ (2) and $\mathrm{Ge}$ (3) on the pressure in reactor.

are in good agreement with the data of [14]. The main, in the realized experimental conditions, are the reactions:

$$
\begin{gathered}
\mathrm{SiCl}_{4}+\mathrm{H}_{2} \rightarrow \mathrm{SiHCl}_{3}+\mathrm{HCl} \\
2 \mathrm{SiHCl}_{3} \rightarrow \mathrm{SiCl}_{4}+\mathrm{SiH}_{2} \mathrm{Cl}_{2} \\
\mathrm{SiHCl}_{3}+\mathrm{H}_{2} \rightarrow \mathrm{Si}+3 \mathrm{HCl},
\end{gathered}
$$

With an increase in pressure above $1 \mathrm{~atm}$., a greater discharge contraction is observed and, at constant power, the discharge ignition stops at a pressure of $1.75 \mathrm{~atm}$. Depending on the pressure (Fig. 1b), an extreme view of the dependence of the yield of trichlorosilane is observed with the maximum at pressure of $0.75 \mathrm{~atm}$. With an increase in pressure above $1 \mathrm{~atm}$., a greater discharge contraction is observed and, at constant power, the discharge stops at a pressure of $1.75 \mathrm{~atm}$. For the process of hydrogen reduction $\mathrm{GeCl}_{4}$, depending on the increase in the ratio of reagents (Fig. 2a) in the initial mixture, the degree of conversion also increases. The main products $\mathrm{GeHCl}_{3}$ and $\mathrm{Ge}$ are formed by the reactions:

$$
\mathrm{GeCl}_{4}+\mathrm{H}_{2} \rightarrow \mathrm{GeHCl}_{3}+\mathrm{HCl}
$$

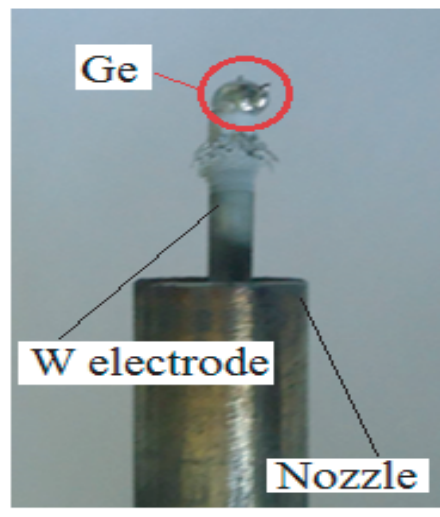

a

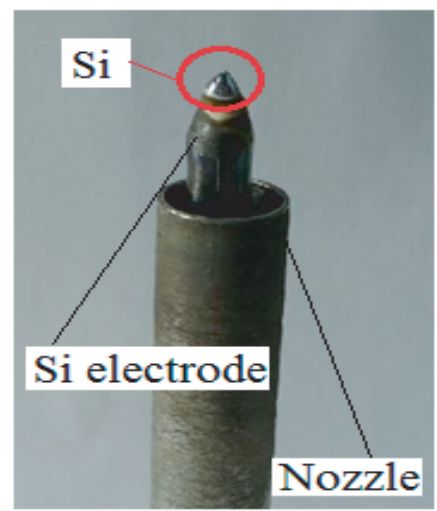

$\mathrm{b}$

Figure 3. View of electrodes after hydrogenation processes reduction of $a-\mathrm{GeCl}_{4}, \mathrm{~b}-\mathrm{SiCl}_{4}$

$$
\mathrm{GeCl}_{4}+2 \mathrm{H}_{2} \rightarrow \mathrm{Ge}+4 \mathrm{HCl},
$$

The dependence of $\mathrm{GeCl}_{4}$ conversion on pressure (Fig. $2 \mathrm{~b}$ ) is not expressed by the maxima. A decrease in $\mathrm{GeCl}_{4}$ conversion and Ge yield is observed while the yield of $\mathrm{GeHCl}_{3}$ increases. The deposition of $\mathrm{Si}$ and $\mathrm{Ge}$ on the electrodes is shown in Fig. 3. It is seen that in both cases the formation of ingot-like, compacted are formed at the ends of the electrodes. Fig. 4 a shows a view of the gas discharge at atmospheric pressure. In the physical experiment, the plasma formation zone is a ball with a radius of $\sim 7 \mathrm{~mm}$, slightly offset to the upper surface of the reaction chamber due to the fact that the output of the reacted gas mixture is located on top. This zone, according to the simulation (Fig. $4 \mathrm{~b})$, is in the temperature range $900-1700 \mathrm{~K}$. The temperature value of $1700 \mathrm{~K}$ corresponds to the area of contact of the electrode with the plasma. It is in this area, as well as in the area surrounding it, during the experiment the deposition of silicon and germanium is observed. Simultaneously with the deposition, the material melts, resulting in a compacted, ingot-like sample (Fig. 3). 900-1700 K high-temperature zone is also a source of atomic hydrogen which contributes to the reduction of $\mathrm{SiCl}_{4}$ and $\mathrm{GeCl}_{4}$. Fig. 4c, d show an isothermal surface with $T=900 \mathrm{~K}$ corresponding to the plasma region and the streamline of the gas mixture through this surface, respectively. According to the conducted numerical experiments, $95 \%$ of the 

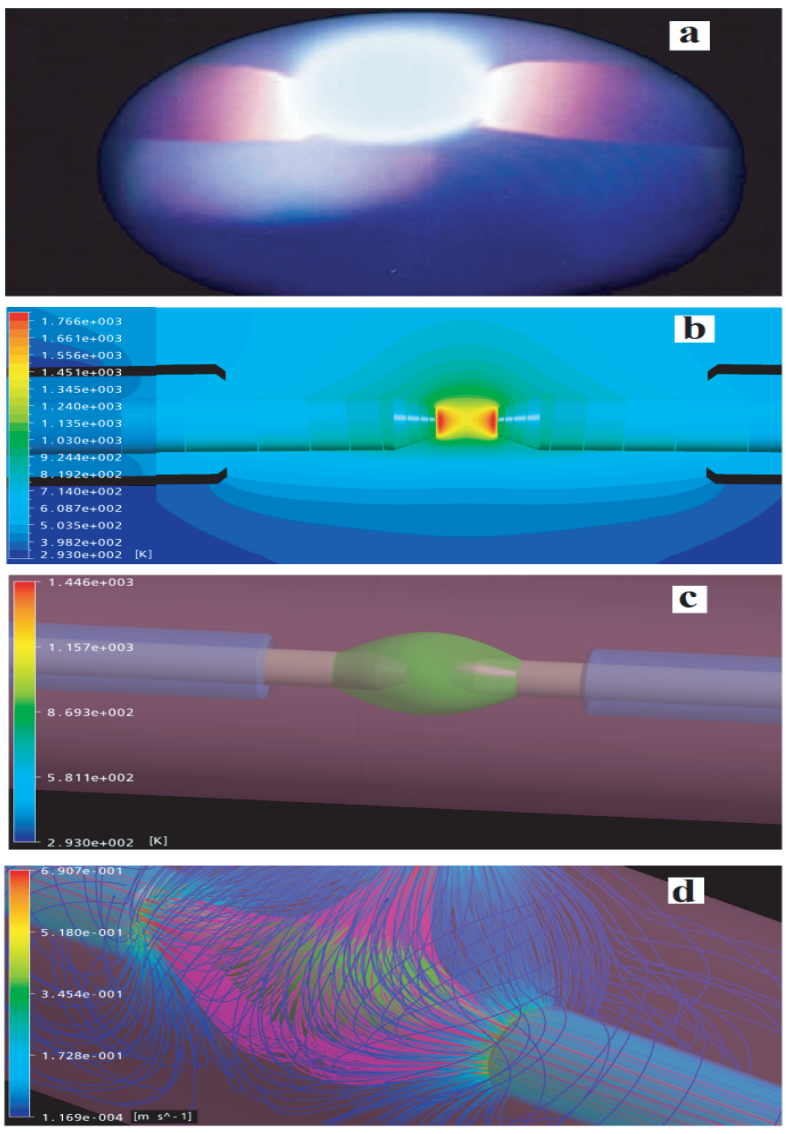

Figure 4. $a$ is the view of gas discharge; $b$ is the distribution of temperature field in plasma zone as well as along the electrode; $c$ is the nozzle-electrode system with isothermal surface $900 \mathrm{~K}$; $d$ are the lines of gas mixture flows in nozzle-electrode-isotherm system $900 \mathrm{~K}$.

gas mixture passes through this surface. Therefore, it can be argued that in the reaction chamber of this configuration there is a full interaction of the gas mixture with the plasma, in which the reaction of reduction of silicon tetrachloride to trichlorosilane takes place. The obtained data on the coefficient of the gas mixture entering the plasma region allow us to state that the yield of trichlorosilane equal to $45 \%$ is close to thermodynamically equilibrium at $T=900 \mathrm{~K}$.

\section{Conclusions}

Under the conditions of RF $(40.68 \mathrm{MHz})$ of a contracted arc discharge of atmospheric pressure, hydrogen reduction processes of silicon and germanium chlorides are investigated. The main products of the hydrogen reduction process are $\mathrm{Si}, \mathrm{Ge}, \mathrm{SiHCl}_{3}, \mathrm{SiH}_{2} \mathrm{Cl}_{2}$ and $\mathrm{GeHCl}_{3}$. Based on the results obtained using numerical simulation, it can be concluded that the temperature of the reaction area of a chemically active plasma is $900-1700 \mathrm{~K}$, and the amount of the gas mixture passing through this region is close to $100 \%$. In this case, the formation of condensed phase is observed at the ends of the electrodes at a temperature close to $1700 \mathrm{~K}$, and the formation of chlorosilanes and chlorogermanes at a temperature of about $900 \mathrm{~K}$.

\section{Acknowledgements}

This work was supported by the grant of the Russian Science Foundation No. 17-13-01027.

\section{References}

[1] Y. P. Raizer. Gas Discharge Physics. Springer, Berlin, 1991.

[2] B. R. Greene et al. Characterization of a $50 \mathrm{~kW}$ inductively coupled plasma torch for testing of ablative termal protection materials. In 55-th AIAA Aerospace Sciences Meeting, number P.0394, 2017. doi:10.2514/6.2017-0394.

[3] B. Giesen et al. Formation of Si-nanoparticles in a microwave reactor: Comparison between experiments and modelling. Journal of Nanoparticle Research, 7(1):29-41, 2005. doi:10.1007/s11051-005-0316-z.

[4] V. Frolov et al. Experimental investigations of the hybrid plasma torch with reverse vortex stabilization. Romanian Journal of Physics, 56:36-40, 2011.

[5] L. V. Shabarova, R. A. Kornev, and P. G. Sennikov. Simulation of gas-dynamic and thermal processes in vortex-stabilized, inductively coupled argon-hydrogen plasma. High Energy Chemistry, 52(5):423-428, 2011. doi:10.1134/S0018143918050120.

[6] D. V. Ivanov and S. G. Zverev. Mathematical simulation of processes in ICP/RF plasma torch for plasma chemical reactions. IEEE Transactions on plasma science, 45(15):3125-3129, 2017. doi:10.1109/TPS. 2017.2773140.

[7] R. A. Kornev, P. G. Sennikov, and A. I. Shishkin. Preparation of boron carbide from $\mathrm{BF}_{3}$ and $\mathrm{BCl}_{3}$ in hydrogen plasma of arc RF discharge. Plasma Chemistry and Plasma Processing, 37(4):997-1008, 2017. doi:10.1007/s11090-017-9821-y.

[8] R. A. Kornev, V. M. Vorotyntsev, A. N. Petukhov, and et al. Catalytic effects of electrode material on the silicon tetrachloride hydrogenation in $\mathrm{RF}$-arc-discharge. RSC Advances, 6:99816-99824, 2016. doi : 10.1039/C6RA21023F.

[9] A. V. Gusev, R. A. Kornev, and A. Y. Sukhanov. Preparation of trichlorosilane by plasma hydrogenation of silicon tetrachloride. Inorganic Materials, 42(9):10231026, 2006. doi:10.1134/S0020168506090172.

[10] A. B. Murphy. Transport coefficients of hydrogen and argon-hydrogen plasmas. Plasma Chemistry and Plasma Processing, 20(3):279-297, 2000. doi : 10.1023/A: 1007099926249 .

[11] A. A. Furman. Inorganic chlorides. M.: Chemistry, page 416 (in Russian), 1980.

[12] R. I. Nigmatulin. Dynamics of multiphase media. part 1. M.: Science, page 416 (in Russian), 1980.

[13] F. R. Menter. Two-equation eddy-viscosity turbulence models for engineering applications. The Amer. Inst. of Aeron. and Astron. Journal, 32(8):1598-1610, 1994. doi:10.2514/3.12149.

[14] K. R. Sarma and C. S. Chanley. Hydrogenation method silicon tetrachloride. Patent 4542004 USA, 1985. 